\title{
EAl Endorsed Transactions

\section{Design Approach for CCSDS Complied Command Operation and Frame Operation Procedure for Integrated Spacecraft Checkout}

\author{
Parul Sharma ${ }^{1, *}$, Sangeetha K. ${ }^{1}$ and Vithal Metri ${ }^{1}$ \\ ${ }^{1}$ Spacecraft Checkout Group, U. R. Rao Satellite Centre
}

\section{Abstract}

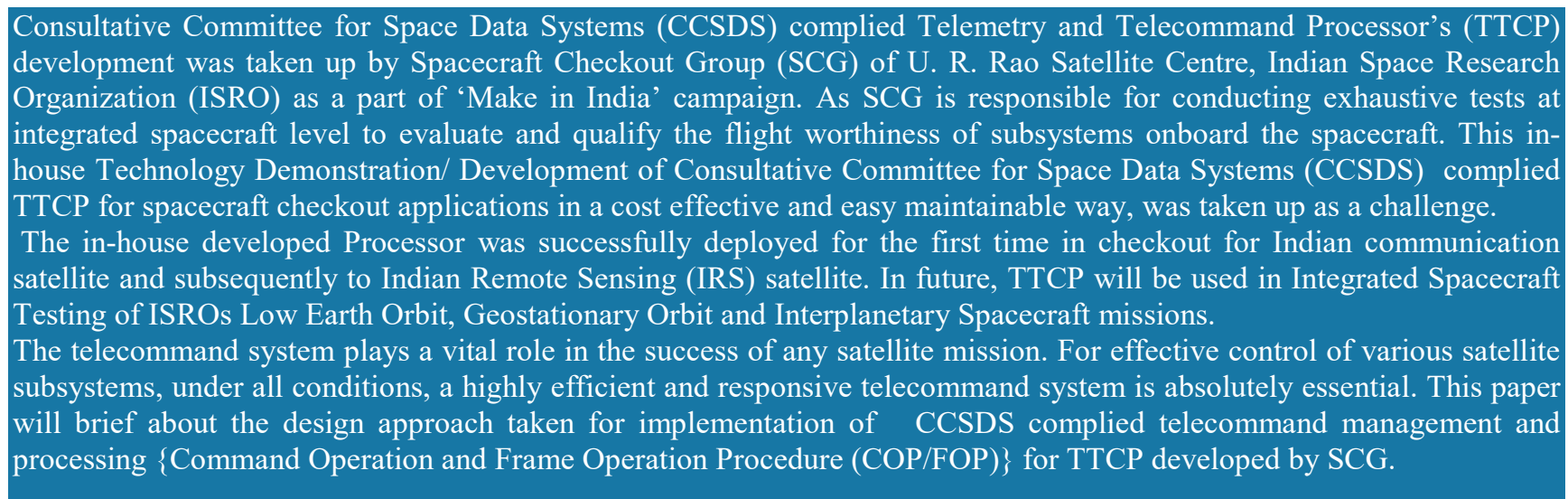

Keywords: CCSDS, TTCP, COP, FOP

Received on 15 October 2018, accepted on 22 November 2018, published on 07 December 2018

Copyright (C) 2019 Parul Sharma et al., licensed to EAI. This is an open access article distributed under the terms of the Creative Commons Attribution licence (http://creativecommons.org/licenses/by/3.0/), which permits unlimited use, distribution and reproduction in any medium so long as the original work is properly cited.

doi: 10.4108/eai.16-5-2018.156532

"Corresponding author. Email:Info11parul@gmail.com

\section{INTRODUCTION}

Spacecraft Checkout Group has developed CCSDS complied Telemetry and Telecommand Processor (TTCP) to support Checkout requirements of ISROs the subsystems onboard along with measurement/experimental data. Telecommand are the commands used to control Communication and Remote Sensing Satellites. Telemetry and Telecommand is a means of communication with satellite. Telemetry gives the overall health of all spacecraft operations remotely through ground checkout (during testing) or ground stations (during mission). The TTCP system developed, is very cost effective and it supports functionalities pertaining to Spacecraft Checkout requirements.

The system comprises of separate telemetry and telecommand hardware units which are interfaced to the external world through a computing server as shown in figure

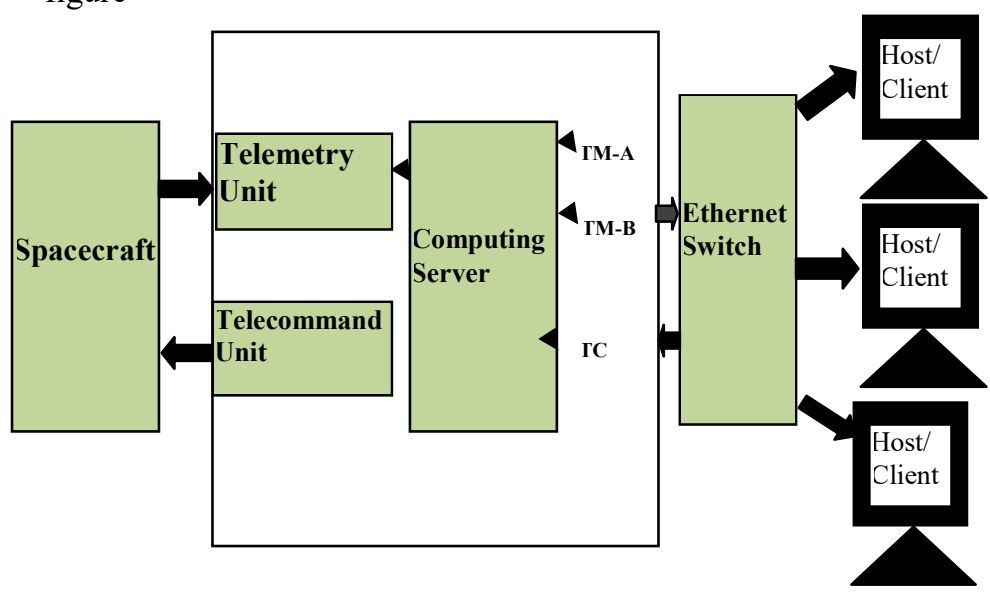

Figure 1. Overall block diagram and checkout interface of TTCP 
The Consultative Committee for Space Data Systems (CCSDS) is an organization established by the member space agencies. ISRO involves as an observer agency for CCSDS. CCSDS defines a standard set of protocols to be followed. CCSDS Telecommand (TC) protocols are uniform standard across globe. CCSDS promotes interoperability and cross-support among cooperating space agencies. CCSDS supports three ways of commanding:[3]

1. BD frames: BD stands for Bypass Acceptance Check and Data. BD frames will get transmitted only once.

2. Link Control Word (CLCW : from Telemetry) is not as expected.

3. BC frames: BC frames involves transmission of control commands which causes a well defined action onboard, which is then reflected in CLCW.

In addition, Directive Requests can be used for further controlling and managing Telecommand operations.

CCSDS based telecommand scheme defines COP and FOP at ground segment whereas Frame Accept and Reject Mechanism (FARM) at space segment. COP is a telecommand protocol which generates, packetizes and transmits the commands to Telecommand Hardware Unit which in turn modulates the command and send it to spacecraft. At onboard, the frames are managed by FARM

Based on Communication Link Control Word received from TM Hardware Unit and the current state of COP, FOP will decide whether command has reached successfully onboard or it requires retransmission in case of unexpected behavior / failure.

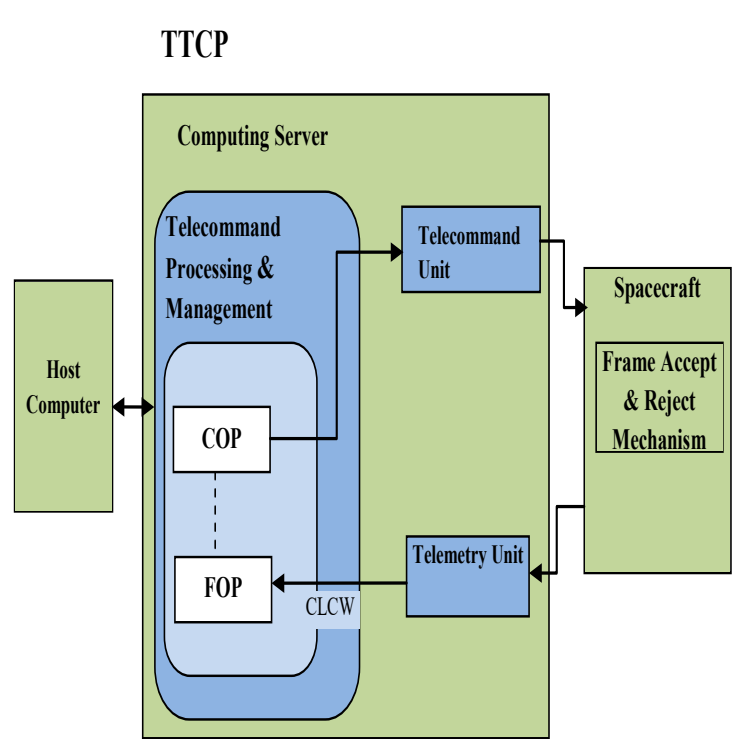

Figure 2. COP, FOP and FARM

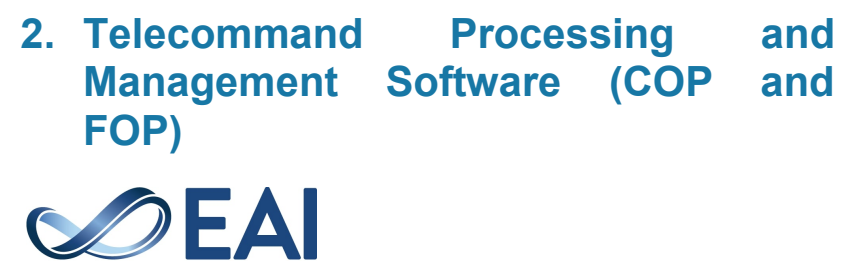

The best software design approach is to separate the software package into functionally independent modules that can be developed in parallel and to define the function of each as well as interfaces between various modules. The same approach is taken in architecting Telecommand Processing and Management Software. The software is decomposed into five major functional blocks [4]:

- TC Processing and Management Control

- TC Processing and Management Monitor

- TC Frame Generator \& Transmitter

- Bypass CLTU generation

- $\quad$ Log \& Display

- $\quad$ TC Echo

\subsection{TC Processing and Management Control}

The purpose of this block is to program functional parameters like Spacecraft id, Virtual Identifiers, CLCW offset in Telemetry etc. All this information will be used for telecommand processing and management. The parameters can be programmed remotely through host computer or through client GUI. Control block will support only one client at a time.

\section{TTCP Telecommand Processing \&Management Overview}

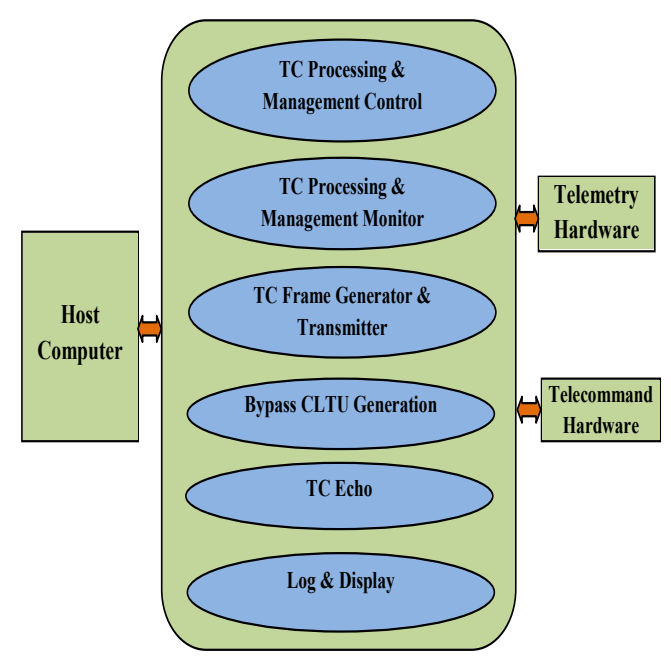

Figure 3. Functional blocks of TTCP Telecommand and Management Software

\subsection{TC Processing and Management Monitor}

The purpose of TTCP TC processing and management monitor is to return back the parameters set using control block. This block also validates whether control parameters are set properly. This block monitors COP configurable parameters only and is manly used by checkout personnel. Therefore, client access is restricted to 4 clients at a time. 


\subsection{TC Frame Generator and Transmitter}

TC frame generator and transmitter implements the layered architecture of CCSDS as shown in figure-4. [2]

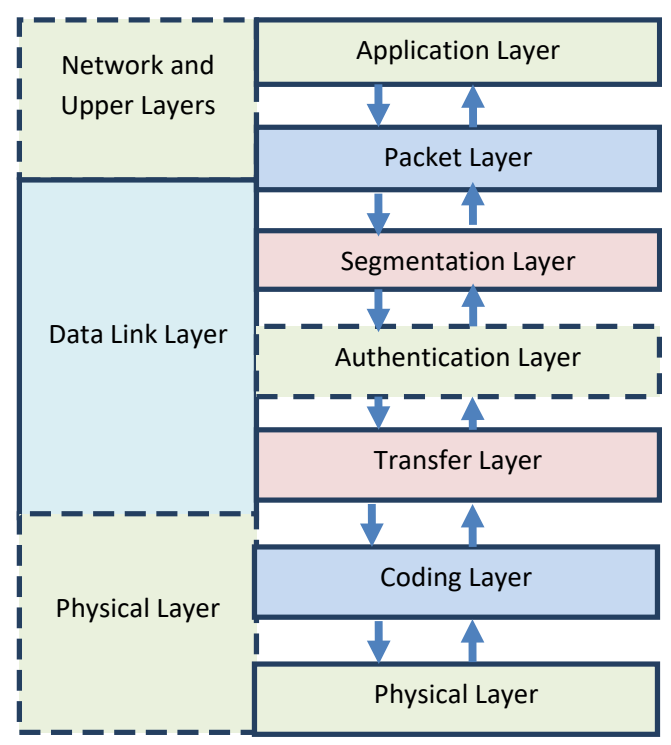

Figure 4. CCSDS Layered Architecture

The above figure-4 shows CCSDS layers which are implemented by TC frame generator and transmitter.

TC frame Generator and Transmitter is the main functional block of Telecommand Processor. This block receives requests from host, validates the request, generates Communication Link Transfer Unit (CLTUs), packetizes and transmits it to Telecommand Hardware. In return, it will receive acknowledgement from hardware, extracts it and gives response to the host. In addition to it, for $\mathrm{AD}$ and $\mathrm{BC}$ mode of commanding, the processor gets CLCW from Telemetry hardware, it extracts CLCW and based on the state and extracted information, it generates Positive/ Negative or Alert response to the host as shown in figure-5.

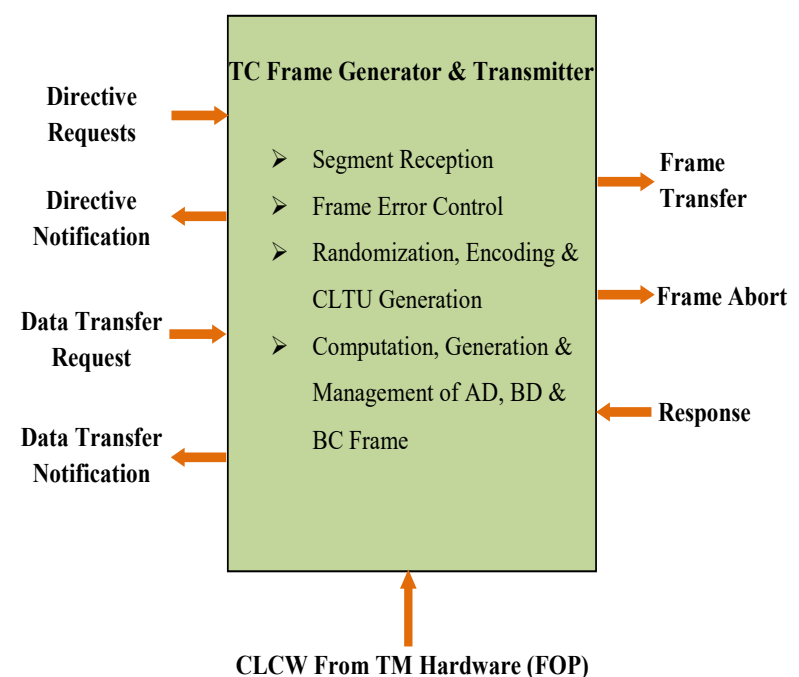

Figure 5. Interfaces of TTCP TC Frame Generator and transmitter
In order to simplify the entire process TTCP TC Frame Generator and Transmitter is further subdivided into following sub-blocks as shown in figure-6. Each of these sub blocks are explained further in detail.

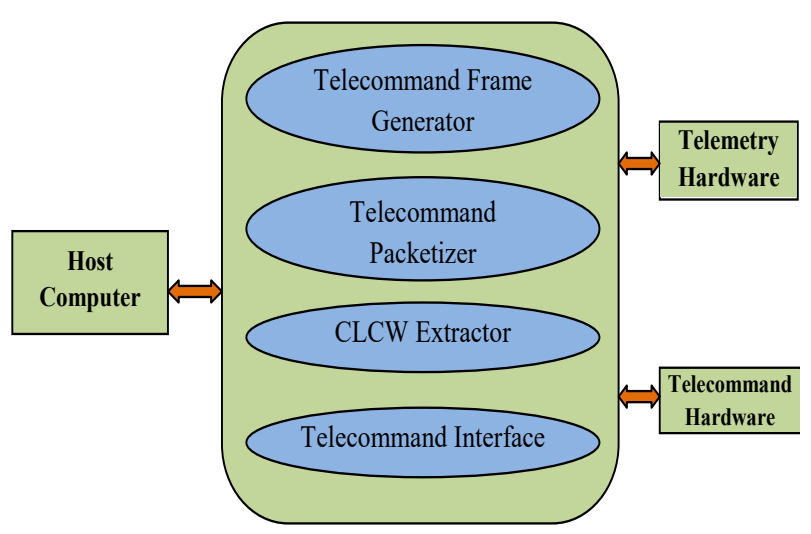

Figure 6. TTCP TC Frame Generator \& Transmitter subblocks

\subsubsection{TC Frame Generator:}

This sub block includes modules to generate Communication Link Transfer Unit ( CLTU ).

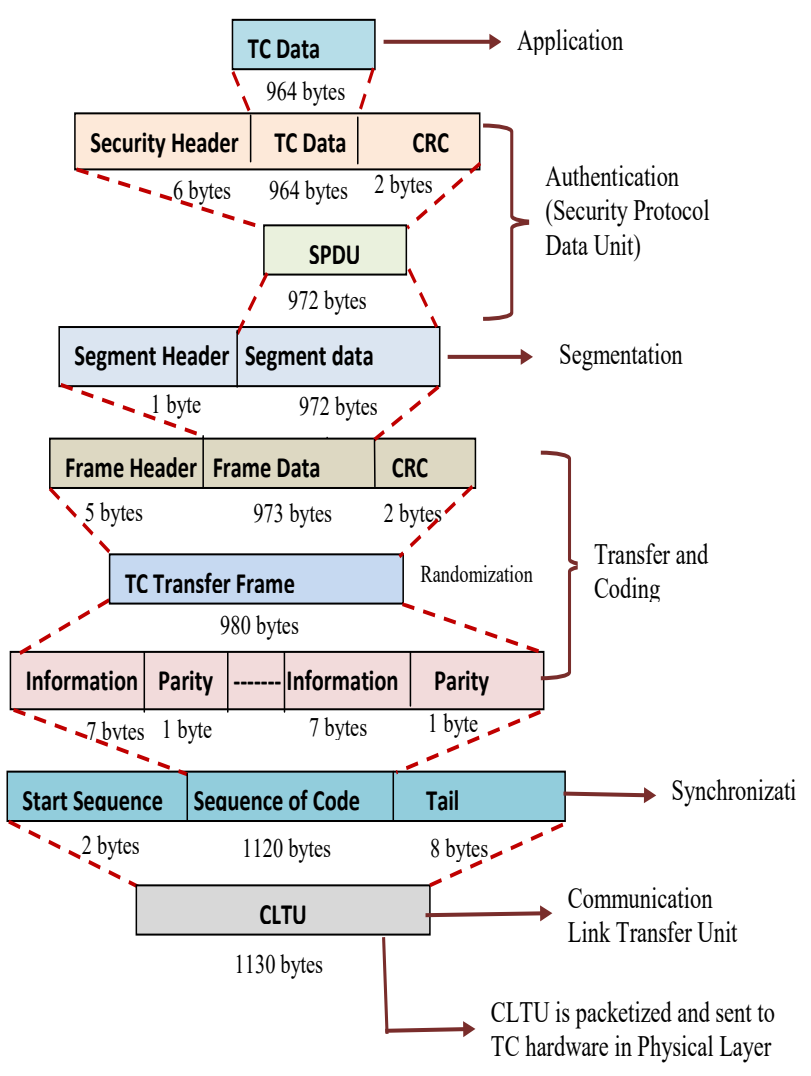

Figure 7. TC Frame Generation Sequence

Commands can be sent in encrypted mode or clear mode. Based on the criticality command transmission mode is 
chosen. TC frame generator supports both encrypted mode and clear mode. Frames are generated by calculating frame header, Cyclic Redundancy Check (optional) is carried out on the data, data together with header and CRC is known as transfer frame. Transfer frame is further randomized and divided into various sub blocks, parity is computed on each sub block and full packet is synchronized by start and end sequence. Detailed frame generation sequence is shown in figure-7.

\subsubsection{TC Packetizer :}

TC Packetizer consists of modules to generate commands packets, various requests and responses.

\subsubsection{CLCW Extractor:}

This module extracts various flags, frame sequence number information required for $\mathrm{AD}$ mode. Whenever AD/BC mode directives are issued, TTCP TC Processing and Management Software will request for Communication Link Control Word (CLCW) from TM Hardware. Various flags and report values will get extracted for processing $\mathrm{AD}, \mathrm{BC}$ mode of commands.

\section{CLCW consists of following information:}

- Control Word Type - 1 bit

- CLCW Version Number - 2 bits

- $\quad$ Status field - 3 bits

- $\quad$ COP in effect -2 bits

- VCID - 6 bits

- $\quad$ Reserved Space - 2 bits

- No RF Available - 1 bit

- No bit lock - 1 bit

- Lockout - 1 bit

- Wait - 1 bit

- Retransmit - 1 bit

- FARM-B Counter - 2 bits

- Reserved Space - 1 bit

- $\quad$ Report Value -8 bits

\subsubsection{TC Interface:}

This block establishes and handles connections with host computer, TC and TM hardware. It calls required modules from various blocks of TTCP Telecommand Frame Generator and Transmitter to handle BD, AD, BC frames and directives. TTCP TC Processing and Management software has 6 states. Each request from host will be validated, processed as per state of TC Processor[3]

- Active (S1) : This is a normal state where there are no recent errors and flow control problems.

- Retransmit Without Wait (S2): This state describes that 'Retransmit' flag is 'on' in CLCW but there are no exceptional circumstances.

- $\quad$ Retransmit with Wait (S3): Software enters in this state if 'wait' flag is 'on' in CLCW.
- Initializing without BC Frame (S4): TC Processing and Management software is in S4 state after receiving Initiate $\mathrm{AD}$ Service (with CLCW check) directive while in Initial state. A successful CLCW check will result in a transition to $\mathrm{S} 1$.

- Initializing with BC Frame (S5): The software is in $\mathrm{S} 5$ state after receiving $\mathrm{BC}$ directives. A successful transmission of $\mathrm{BC}$ request and a clean CLCW will result in transition of $\mathrm{S} 1$.

- Initial (S6): Initial state is the first state of TC Processor.

The state-event is very large and the state transitions are very complex. The software designed, has the capability to handle state transitions automatically. The state transition occurs based on the telemetry reception from Telemetry Unit. The CLCW is extracted from the received telemetry frames, the data is processed which initiates event occurrence (event numbers are well defined in CCSDS protocol) and based on event and current state of TC Processor, decision of state transition is carried out. A sample state transition diagram is shown below:

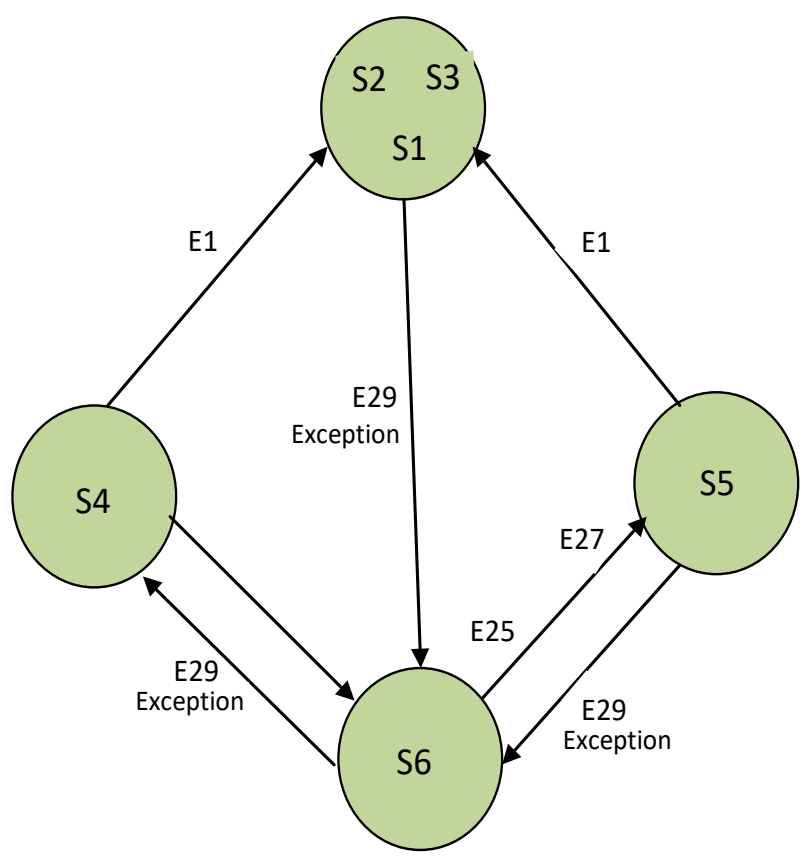

Figure 8. A Sample State Transition Diagram

Table 1. Event Description

\begin{tabular}{|l|l|}
\hline $\begin{array}{c}\text { Event } \\
\text { Number }\end{array}$ & \multicolumn{1}{c|}{ Event Details } \\
\hline & $\begin{array}{l}\text { CLCW arrives in form of valid COP-1 Pattern } \\
\text { as: }\end{array}$ \\
E1 & $\begin{array}{l}\text { Lockout Flag }=0, \\
\mathrm{~N}(\mathrm{R})=\mathrm{V}(\mathrm{S}), \\
\text { Retransmit Flag }=0, \\
\text { Wait Flag }=0, \\
\mathrm{~N}(\mathrm{R})=\mathrm{NN}(\mathrm{R})\end{array}$ \\
& Receive Directive From Management Function \\
\hline E25 & \\
\hline
\end{tabular}




\begin{tabular}{|l|l|}
\hline $\begin{array}{c}\text { Event } \\
\text { Number }\end{array}$ & \multicolumn{1}{c|}{ Event Details } \\
\hline & $\begin{array}{l}\text { Initiate AD Service (with CLCW check) } \\
\text { Directive, } \\
\text { BC_Out_Flag = Ready }\end{array}$ \\
\hline E27 & $\begin{array}{l}\text { Receive Directive From Management Function } \\
\text { Initiate AD Service (with Set V(R) Directive) } \\
\text { BC_Out_Flag = Ready }\end{array}$ \\
\hline E29 & $\begin{array}{l}\text { Receive Directive From Management Function } \\
\text { Terminate AD Service Directive }\end{array}$ \\
\hline
\end{tabular}

$\mathrm{N}(\mathrm{R})=$ Frame Sequence Number in CLCW

$\mathrm{NN}(\mathrm{R})=$ Next expected frame sequence number

$\mathrm{V}(\mathrm{S})=$ Transfer frame sequence Number

\subsection{ByPass CLTU Generation:}

This block directly transmits the command to TC Hardware bypassing all the processing related to CLTU generation.

\subsection{Log \& Display:}

This block deals with the logging and display of commands. Logging of commands will be done along with CLTU and the commands issued from host computer. Time reference is also logged for each command. Display will show the transmission status, date and time and the command code in GUI for quick reference.

\subsection{TC Echo:}

TC Echo block is used to dump either CLTUs or Transfer frame based on the request by clients. During Spacecraft IST, there is a requirement to provide command to different agencies involved during testing. To meet this requirement provision is provided to support 8 clients.

\section{Testing the Telecommand interface}

Testing is the process of evaluating a system or its components with the intent to find whether the product satisfies the specified requirements or not. Testing is executing a system in order to identify any gaps, errors or missing requirements in contrary to actual requirement, Before communicating with the spacecraft thorough testing is carried out to clear the interface.The software was subjected to a third party team. Various test cases were generated which includes testing of variable length commands, continuous commanding, verification of frame transmission to multiple clients, abrupt disconnection of clients, abrupt closure of application, abrupt network failures etc . Interface testing of TTCP was also carried out with onboard computer. A proper plan was worked out to clear $\mathrm{BD}, \mathrm{BC}$ and $\mathrm{AD}$ frame execution onboard. A gist of plan is as follows:

- Configure the host system for BD frames

- Initiate commanding to onboard decoder and verify the reception
- Configure onboard for AD frames by sending BC commands

- Unlock the onboard receiver through BC directives

- $\quad$ Synchronize onboard and ground by setting frame sequence number directive

- Configure the host system for AD frames

- Initiate commanding to onboard decoder and verify the reception

Thus, exhaustive testing was carried out to ensure satisfactory results before deploying it to various satellite projects .

\section{CONCLUSION}

The successful implementation of CCSDS based Telecommand Processing and management software for in-house developed TTCP has resulted in significant cost reduction for checkout operations. Costly procured TTC Processor Units for both CCSDS Telecommanding and Telemetry reception, were in use prior to the deployment

of indigenous unit in Checkout. In addition, the cost for

Annual Maintenance of the procured units is significantly high. Having the TTCP units, designed and developed inhouse, the required expertise and resources are readily available for future requirements and maintenance. In future, the software will be upgraded for commanding in both CCSDS and Non-CCSDS based projects. Moreover, various possibilities of deploying it for ground stations can also be explored.

\section{Acknowledgment}

We express our gratitude to Dr. Hariharan V.K. Deputy Director, Integration and Checkout Area and Smt. Vasantha Kumari U. N. Group Director, SCG for their inspiring motivation and guidance. We are thankful to Smt. Usha Bhandiwad Group Head, SCG for constant support in completing the paper.

\section{References}

[1] CCSDS BLUE BOOK 232.0-B-1 (TC Space Data Link Protocol)

[2] CCSDS BLUE BOOK 231.0-B-1(TC Synchronization And Channel Coding)

[3] CCSDS 202.1-B-1 (Command Operation Procedure)

[4] Software Design Document for TTCP Server Software 